\title{
Computational study of active flow control drag reduction device for utility vehicle
}

\author{
Jędrzej Mosiężny ${ }^{1, *}$ and Bartosz Ziegler², \\ ${ }^{1}$ Redos Trailers Sp. z o.o. Kolejowa 33A, 64-300 Nowy Tomyśl, Poland \\ ${ }^{2}$ Poznań University of Technology, Chair of Thermal Engineering, Piotrowo 3, 60-965 Poznań, Poland
}

\begin{abstract}
The study presents a computational study of a drag reduction device based on an active boundary layer control for a generic truck-trailer utility road vehicle. The conceptual device is in accordance with upcoming EU regulations regarding attachable aerodynamic devices for heavy utility vehicles. Design and principles of operation of the conceptual device are presented. The device is intended to increase decrease the trailer's base drag coefficient by manipulation of the separated flow region behind the vehicle base. Results of a steady state Reynolds averaged analysis and Delayed Detached Eddy Simulation are presented to show the discrepancies of fluid flow patterns between baseline and augmented configuration as well as between mentioned CFD approaches. Results for drag reduction for baseline truck-trailer configuration and aerodynamically augmented configuration are presented.
\end{abstract}

\section{Nomenclature}

\subsection{Abbreviations}

AFC - Active Flow Control

CFD - Computational Fluid Dynamics

DDES - Delayed Detached Eddy Simulation

LES - Large Eddy Simulation

RANS - Reynolds Averaged Navier-Stokes

S-A - Spalart-Allmaras (turbulence model)

SST - Shear Stress Transport (turbulence model)

TKE - Turbulence Kinetic Energy

\subsection{Symbols}

$\mathrm{C}_{\mathrm{D}}$ - Drag coefficient

$\mathrm{D}$ - Drag force

$\mathrm{L}$ - turbulence integral length scale

$\mathrm{k}$ - Turbulence kinetic energy

$\beta *$ - SST model constant

$\varepsilon$ - Rate of dissipation of turbulence energy

$\omega$ - Turbulence specific dissipation rate

$\mathrm{x}, \mathrm{y}, \mathrm{z}$ - Cartesian coordinates

\section{Introduction}

This study relates to modelling flow fields of heavy road vehicles. It focuses on predicting drag forces of a generic truck-trailer configuration road vehicle, as well as modelling flow fields of vehicle augmented with active flow control (AFC) devices aimed at reducing the vehicle pressure drag. Research in this field is recognized as being

*e-mail: jedrzej.mosiezny@ @ut.poznan.pl an important part of economic and environmental policies $[1,2]$. As a result a directive was introduced by European Union [3], part of which introduces regulations allowing aerodynamic devices protruding by a limited distance outside maximum legal vehicle length. This created a need for aerodynamic devices capable of creating substantial aerodynamic forces with limited surface area. AFC surfaces are suitable for such applications, as in the past they showed aerodynamic loadings far beyond limits for passive devices $[4,5]$ Especially circulation control by steady tangential blowing in the boundary layer region seems promising for such applications. For design purposes of such devices, the precise value of vehicle drag is not of greatest importance, as much more important is the incremental drag prediction (difference in drag force between configurations) as it is the parameter driving the design process. It is also important to predict accurately the momentum exchange between truck/trailer boundary layer and jets introduced by AFC device. Computational analysis should be therefore focused on possibly accurately capturing flow physics at trailer base, and in the vicinity of AFC devices, with special efforts to provide solution resolution and accuracy in boundary layer region of drag reduction device. Accurate representation of truck and chassis aerodynamics is of lower priority for the purpose of drag reduction device design and assessment. In this article, analysis of baseline geometry and case with AFC device are presented. Geometries and operational parameters for both cases come from a research grant for drag reduction device R\&D grant "Development of a light trailer construction with reduced aerodynamic drag" of REDOS Trailers Sp. z o. o. subsidized by polish National Center for Research and Development. Figure 1 shows the base- 
line geometry of a truck-trailer configuration. Augmented geometry was basically the same as the baseline geometry, with cylindrical Coanda surfaces based on the circulation control wing concept $[4,5]$. As the mentioned research program is ongoing, and the geometries are considered company's proprietary information, detailed geometries and AFC parameters are not provided in this article. The study focuses on CFD methodology and differences between RANS and DDES approaches.

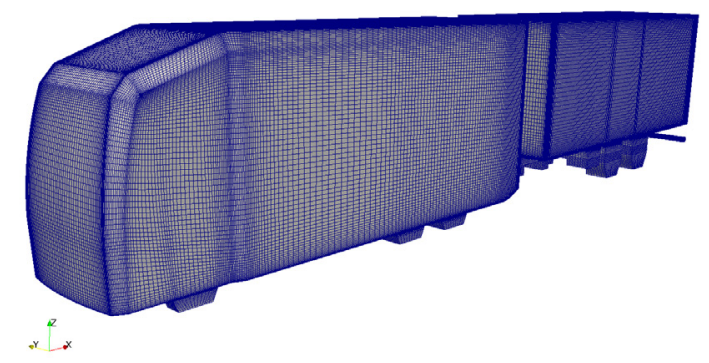

Figure 1. Generic truck trailer configuration with surface mesh

\section{Approach to physical and numerical modeling}

\subsection{Turbulence modeling}

The flow field in question is characterized by a Reynolds number of the order of $3 \cdot 10^{7}$, based on the vehicle length and freestream velocity, it is therefore a fully turbulent flow. The truck/trailer boundary layers arrive at the drag reduction device, which is in general a smooth, curved surface. Flow along that surface experiences significant (both favorable and adverse) pressure gradients because of the imposed aerodynamic loading and then become shear layers of the separated region downstream of the vehicle. For such application, turbulence modelling approach should be focused on the following abilities:

a Accurately resolving sidewall boundary layer profiles of velocity, and turbulence parameters, as those define stream conditions for the drag reduction device.

b Low-Re approach (resolving viscous sublayer, no wall functions) is required, as limits of aerodynamic loading is inherently associated with flow separation.

c Capturing as accurately as possible, momentum transport in free shear layers around trailer base separated region.

Requirements $a$ and $b$, fit to the use of RANS analyses, with one of Boussinesq hypothesis based turbulence models tuned for turbulent boundary layers. Most of 2-equation models from k- family as well as SpalartAllmaras (S-A) [6] model can predict turbulent boundary layer velocity profiles with sufficient accuracy [7,8]. Validation studies show however, that most of them (especially standard k- $\mathrm{E}$ ) cannot predict flow separations from smooth surfaces accurately. Only SST [9] and S-A are able to predict the separation/reattachment with reasonable accuracy (with SST being slightly better), what is specifically shown on cases of axisymmetric and flat flow diffusers $[10,11]$ presented in mentioned references [7, 8, 12].

Requirement $\mathrm{c}$ is much more problematic. For complex flows like the trailer wake, RANS (both steady and unsteady) offers limited accuracy, as the wake separated region is dominated by momentum transport associated with large scale eddies. It is therefore very often assumed that RANS/LES hybrid approaches like DES or DDES are more appropriate for such cases. In this paper, differences in some flow features and resulting drag prediction differences are compared between steady RANS and much more computationally demanding DDES. Despite higher computational cost, the two equation SST model was chosen over the one equation S-A for its higher versatility and reattachment prediction capability [7]. It was used in RANS simulations, as well as a subgrid scale model in DDES simulations. Identical choice of subgrid scale model for both (RANS and DDES) approaches allows also to point out differences from using hybrid RANS/LES approach between analyses clearly.

\subsection{Mesh}

In order to properly resolve the flowfield with accordance to the requirements of the turbulence models in use, a quality mesh, with high resolution of boundary layer is necessary.

First a generic geometry of a modern truck/trailer vehicle is prepared. The geometry is defeatured from items irrelevant from the standpoint of the flow, such as elements of the undercarriage, cab panel dilatation, door handles etc. Chosen model of the truck features no side mirrors, therefore the source geometry for mesh is also lacking the side mirrors. Although all these elements contribute to the overall aerodynamic drag, the geometry of these features is intricate and contributes to a very complicated mesh with enormous number of elements. Geometry of the aerodynamic device was created within the meshing software itself. As the geometrical features of the device are below $1 \mathrm{~mm}$ of size, in comparison to the $18 \mathrm{~m}$ vehicle, creating the geometry in the meshing software decreased the risk of geometry errors coming from translating the CAD files between the systems.

Performed literature studies of truck aerodynamics showed that a common practice for analyzing such cases is using an unstructured mesh with inflation layer or meshes generated by automatic "cut cell" based algorithms. Such approach is comfortable and does not require much work time to generate the grid, with a downside of a grid with hundreds of millions of elements. For presented study, computational resources are limited, therefore this approach was dropped in favor of hand-crafting the meshes.

Two sets of meshes were prepared for the study. Set one consisted of the URANS grids, prepared during the first stage of the R\&D grant, while many concepts of aerodynamic devices were tested by means of CFD. Therefore 
a baseline mesh with capability of interfacing the aerodynamic device was created. Any mesh for conceptual aerodynamic device was saved as a separate project and merged to the aforementioned mesh with interfaces at non matching boundaries. Although comfortable, this solution proved to be numerically unstable and increased time to convergence. Also, unsteady RANS analyses showed further issues with mesh induced calculation instabilities. Meshes for second stage of the grant were rebuilt from the ground up for baseline configuration and a small count of most promising aerodynamic augmentations. New grids, despite the geometrical intricacies of the AFC devices, omit the internal interfaces and address the issues of mesh induced errors encountered during the unsteady RANS stage.

Baseline and augmented mesh represents full (meaning, not half) geometry. Coordinate system orgin is placed at crossing of planes of trailer base, symmetry and ground. Positive $\mathrm{x}$ direction is the direction of the flow, positive $\mathrm{z}$ direction is the normal to ground plane vector.

In order to reduce the number of elements, a mesh with structural, hexagonal nearfield and tetrahedral farfield region is created. Due to complex topology, an area below the trailer base is filled with tetrahedral elements. Non dimensional wall element spacing $(\mathrm{y}+)$ is set to $1(17 \mu \mathrm{m})$ for all surfaces of interest. All wall spacings are computed for $25 \mathrm{~m} / \mathrm{s}$ flow velocity, $18 \mathrm{~m}$ characteristic dimension and thermophysical properties of standard air. The only mesh region with thicker mesh elements in the boundary layer, is the chassis region. This is because this region is dominated by separated regions, and negligible flow velocities close to chassis element walls and creating high resolution boundary layers was considered unjustified in that region. On the chassis first element spacing of $8 \mathrm{~mm}$ was applied (which for freestream values would refer to $\mathrm{y}+$ around 450). During post-processing it was calculated that for most area of chassis elements $y+$ was still below 10 as in that region the flow was considerably slower than around side and top walls.

Tangential wall sizing is set in a following manner. Values of turbulent kinetic energy and turbulence specific dissipation rate are obtained from RANS analyses performed in Stage 1 of the grant. Next, an integral length scale $\mathrm{Lt}$ is obtained from formula 1 [13].

$$
\mathrm{L}_{\mathrm{t}}=\frac{\mathrm{k}^{(3 / 2)}}{\varepsilon}=\frac{\sqrt{\mathrm{k}}}{\beta * \omega}
$$

Based on preliminary RANS calculations, values of $\mathrm{k}=40 \frac{\mathrm{m}^{2}}{\mathrm{~s}^{2}}$ and $\omega=360 \mathrm{~s}^{-} 1$ are obtained for wake shear layers, which corresponds to integral length scale of 195 $\mathrm{mm}$. Source [13] provides that for resolving minimum of $80 \%$ of turbulent energy, it is required that the cell sizing is at most of 0.21 times the $\mathrm{L}_{\mathrm{t}}$ value, resulting in the maximum element edge length of c.a $40 \mathrm{~mm}$. This element sizing is kept in regions where LES behavior is intended, that is in the nearfield (boundary layer directions tangent to wall) and in the wake region behind the trailer base.

Efforts were made to generate grids of highest possible quality possible with special attention to non- orthogonality. Definition of non-orthogonality used for this case is: an angle between a face normal vector and vector connecting the centroids of neighboring elements, with 90 degrees being worst element and 0 degrees being a fully orthogonal (or cartesian) element. Baseline mesh reaches the worst orthogonality of 57 degrees, augmented configuration mesh reaches the worst nonorthogonality of 63 degrees. Final meshes are on range of 20 million element count with 18 million hexagonal cells in the nearfield region. Computational grids are created in Pointwise meshing software. Outlines of the grids are presented in the figure 2 .

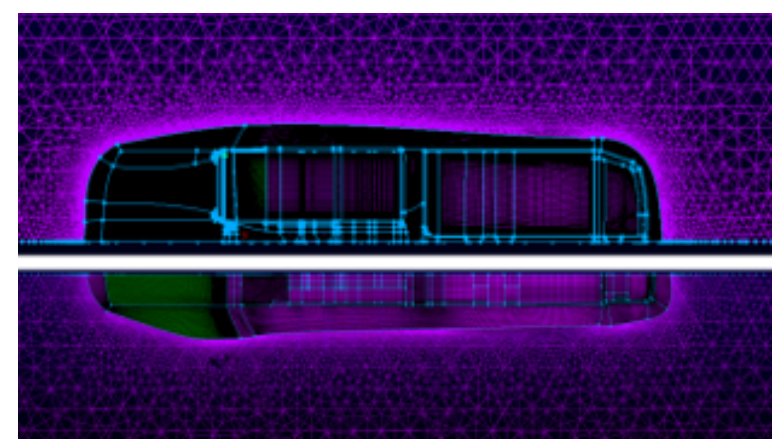

Figure 2. Outline of the mesh. Top - symmetry plane, bottom ground view

\subsection{Numerical setup}

RANS and DDES analysis are performed on the same numerical setups, with the only difference being the turbulence strategy and time domain discretization. Gradient discretization is Least Squares Cell Based, momentum equations are discretized with Bounded Second Order Differencing. Second order discretization is applied to pressure, turbulent kinetic energy and specific dissipation rate equations. Time discretization is set to Bounded Second Order Implicit. Timestep size for DDES calculations is set to 1e-04 seconds, which corresponds to volume averaged Courant numbers of 0.01 , with local maxima below 16. This time step gave sufficient CFL numbers in regions of LES behavior (below 0.05) for temporal resolution and achieved close to optimal flowtime to walltime relation. Total flowtime is set to 2.2 seconds.

The domain inlet is set to 'velocity-inlet' type boundary condition with velocity magnitude of 25 [m/s]. Domain outlet is a pressure outlet type boundary condition with $0[\mathrm{~Pa}]$ outlet pressure value. Boundary representing the road is a viscid wall and farfield boundary is set as inviscid wall. Both ground and farfield walls have relative linear motion enabled to simulate the movement of the vehicle with relation to the ground. Multiple farfield boundary condition combinations were tested, including a pressure outlet and incompressible far-field BC at the farfield boundary, with inviscid moving wall being the most reliable. Working fluid is an incompressible standard air with properties corresponding to ISA $15^{\mathrm{O}} \mathrm{C}$ air properties at sea level. The domain pressure reference is set to $101325[\mathrm{~Pa}]$. 


\subsection{Solution strategy}

The flowfield is initialized using a Fast Multigrid algorithm with setup identical to the main calculation. This operation initializes the flowield with velocity and pressure field close to a converged solution. Next a steady state RANS analysis is performed. Target weighted residual criterium was set to 1e-10. Both cases reached the residual level below 1e-03 after 15000 iterations and reached a plateau. After that, no significant changes occurred in the flowfield. The chassis flow still experienced some oscillations but with no influence on the calculated drag by any meaningful value. It must me noted that the goal for the RANS analysis is not to reach a fully converged solution, but to resolve the flow field with developed boundary layer and wake. Earlier, (yet unpublished) studies showed, that launching a DDES analysis from a well converged steady state solution increases the number of timesteps required to induce DES type flowfield. It is decided to use an not converged RANS solution to star the DDES analysis.

The DDES analysis is set to run for 22000 timesteps, which corresponds to 2.2 seconds of flowtime. At $25[\mathrm{~m} / \mathrm{s}]$ velocity and total vehicle length of $18 \mathrm{~m}$ for baseline and $18.5 \mathrm{~m}$ for augmented configuration, the time which the freestream passes the full length of the vehicle is about 0.7 seconds. It is decided that first 0.7 second "passthrough" is the intermittent analysis, where characteristic for DDES analyses flow patterns are generated from the Reynolds Averaged flow field. Following two "passthrough" times of $1.4 \mathrm{~s}$ are considered as the data acquisition time, where a coefficient of drag is computed and stored for each timestep.

\section{RESULTS AND DISCUSSION}

\subsection{Flowfield comparisons}

Following control surfaces are included in the comparison of the flowfield: symmetry plane of the vehicle and two normal to ground planes at $2 \mathrm{~m}$ and $0.15 \mathrm{~m}$ distance from the ground respectively. Contours of velocity magnitude, static pressure and turbulent kinetic energy are compared between RANS and DDES cases. Figures 3 and 4, show comparison of velocity magnitude at $\mathrm{z}=2 \mathrm{~m}$ and $\mathrm{z}=$ $0.15 \mathrm{~m}$ (chassis flow) for the baseline case (Steady RANS - top and DDES at time instance of $1.8 \mathrm{~s}$ - bottom). At the front of the vehicle, the differences are minor, even the side separations are of similar extent. Downstream of them however, flowfields differ substantially, as the averaged solution exhibits typical shear layer, with velocity gradient perpendicular to the main flow direction (x axis). DDES solution has that region dominated by large scale vortices and the velocity field is more nonuniform. This is consistent with fields of turbulence kinetic energy (Figure 5 - note the logarithmic scale). Differences between RANS and DDES approaches, are clearly seen on vorticity fields 6 , where RANS shows vorticity only in places of significant shear while distinctive eddies are visible on DDES results, especially in the wake region. Velocity comparisons show that the augmented case separated wake region is dominated by smaller length scale eddies as well as lower average velocity magnitude.
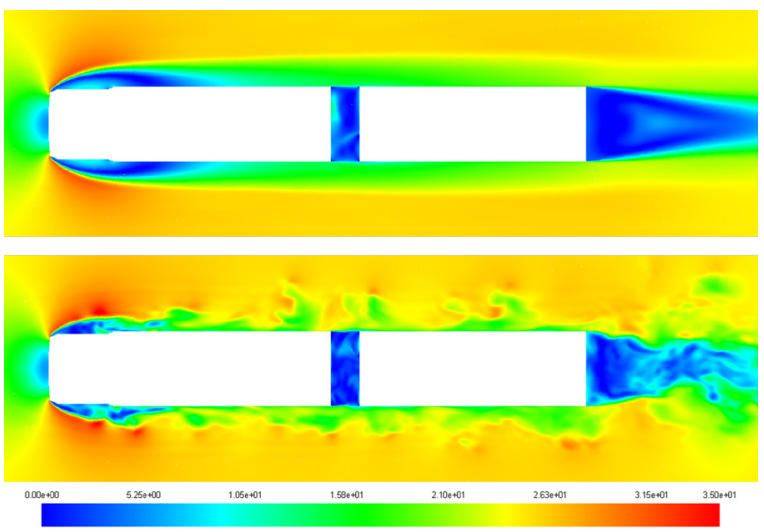

Figure 3. Baseline velocity contour, $z=2 \mathrm{~m}$, upper - RANS, lower-DDES

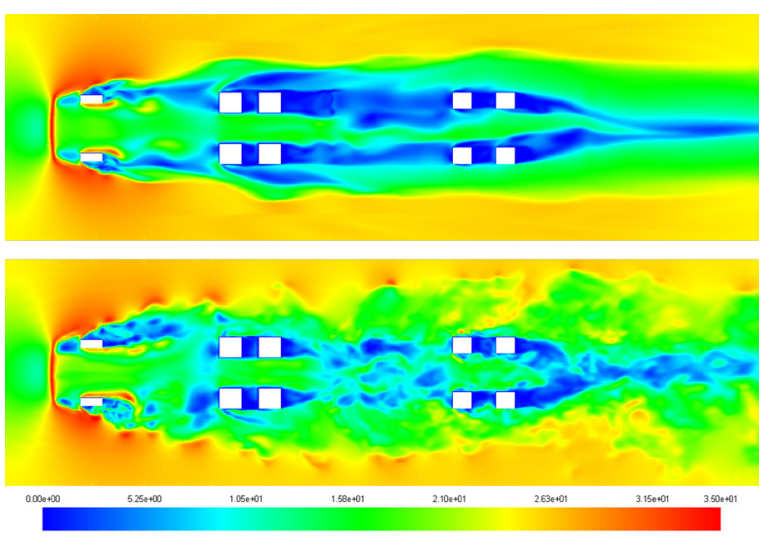

Figure 4. Baseline velocity contour, $\mathrm{z}=0.15 \mathrm{~m}$, upper - RANS, lower - DDES
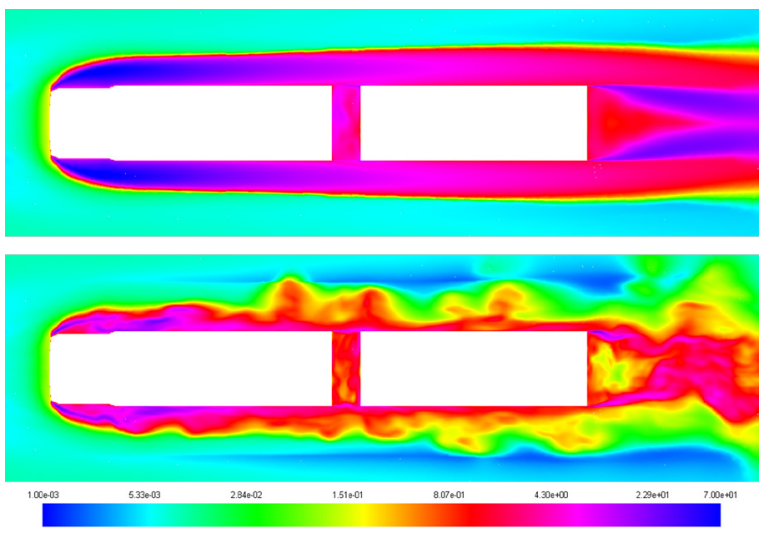

Figure 5. Baseline TKE contour (log scale, $\mathrm{z}=2 \mathrm{~m}$, upper RANS, lower - DDES

\subsection{Drag predictions}

The project goal is to accurately assess the aerodynamic drag reduction of the truck-trailer configuration aug- 


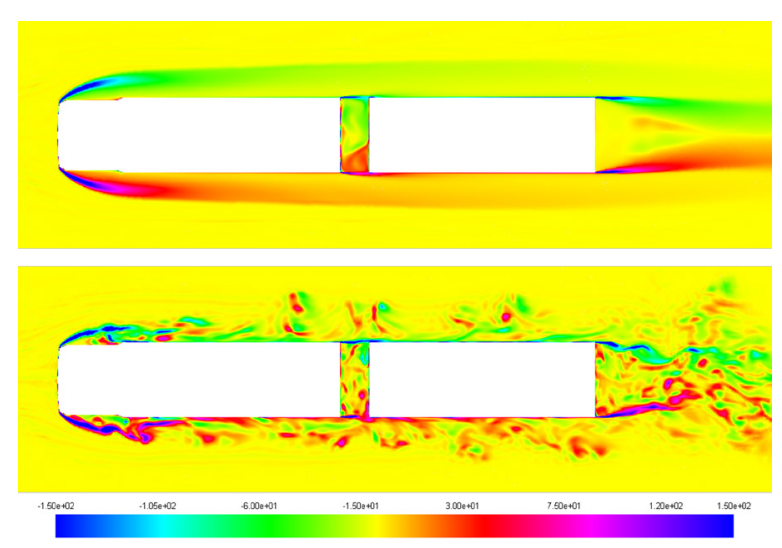

Figure 6. Baseline $\mathrm{z}$-vorticity contour, $\mathrm{z}=2 \mathrm{~m}$, upper - RANS, lower - DDES
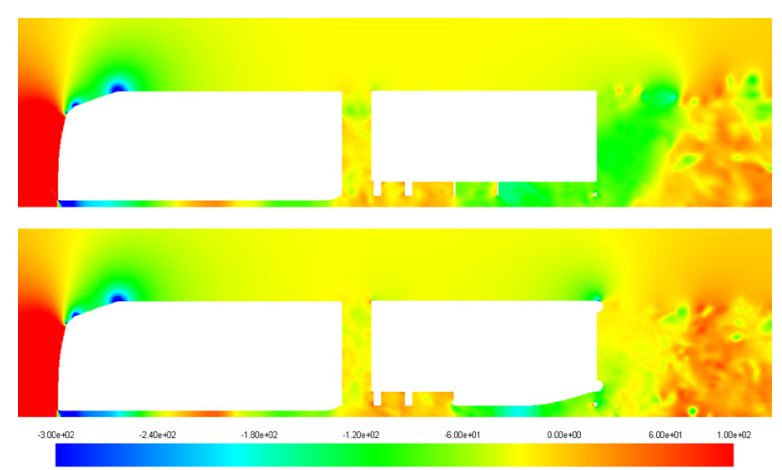

Figure 7. Pressure contour comparison on symmetry plane, upper - baseline, lower - augmented
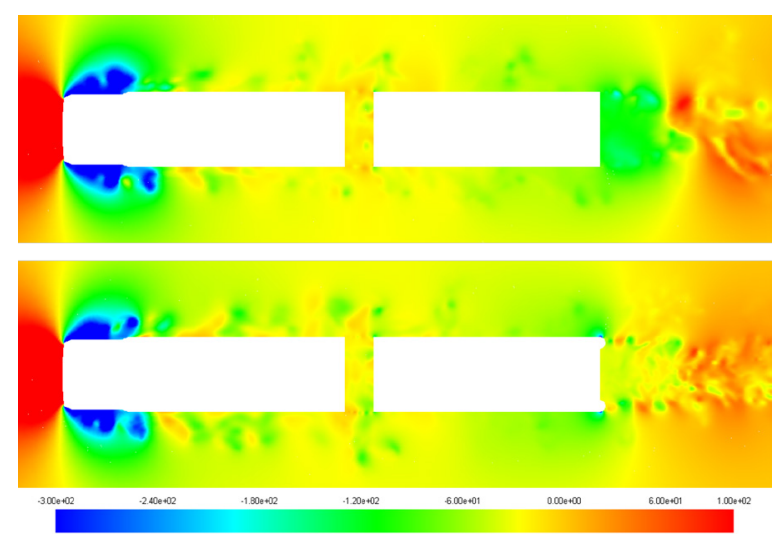

Figure 8. Pressure contour comparison on $\mathrm{z}=2 \mathrm{~m}$ plane, upper - baseline, lower - augmented

mented with an Active Flow Control device. Comparison of drag coefficients is presented in Table 1 (A1 indicates case with AFC device). DDES drag coefficient values are presented for instantaneous time values from $1.8 \mathrm{~s}$ time instance. Time averaged value of CD is also provided. It is seen that augmentation with an Active Flow Control aerodynamic device provides a reduction of aerodynamic drag of $5.85 \%$ for RANS analyses and $15.59 \%$ for time averaged DDES analysis.

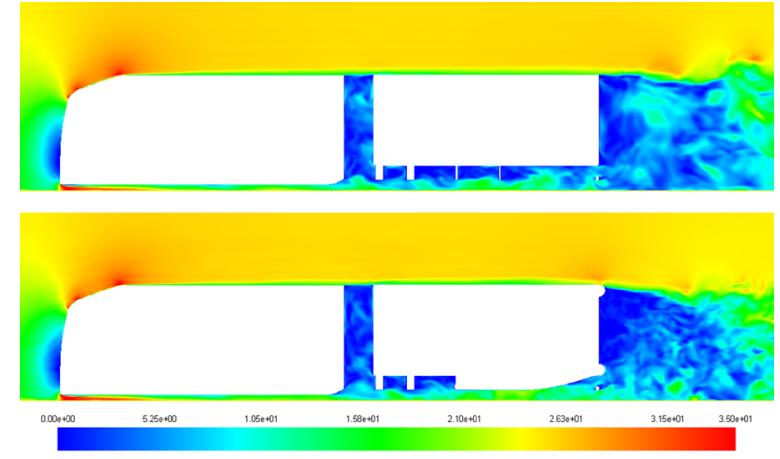

Figure 9. Velocity contour comparison on symmetry plane, upper - baseline, lower - augmented
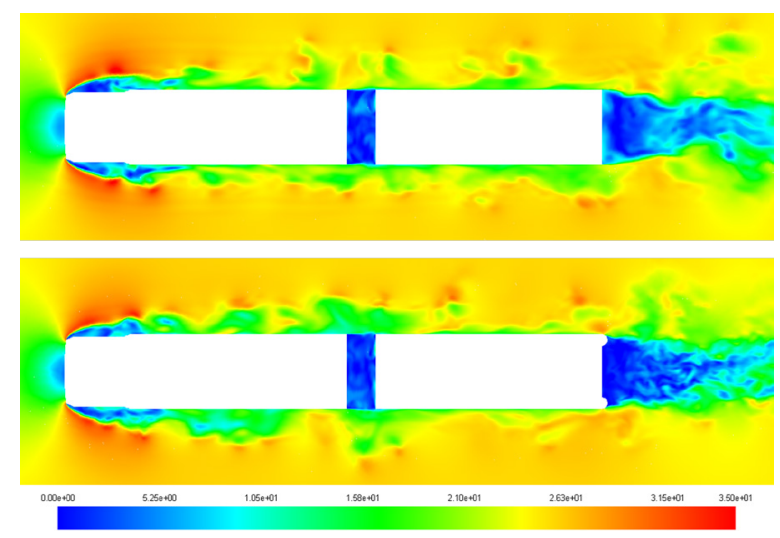

Figure 10. Velocity contour comparison on $\mathrm{z}=2 \mathrm{~m}$ plane, upper - baseline, lower - augmented

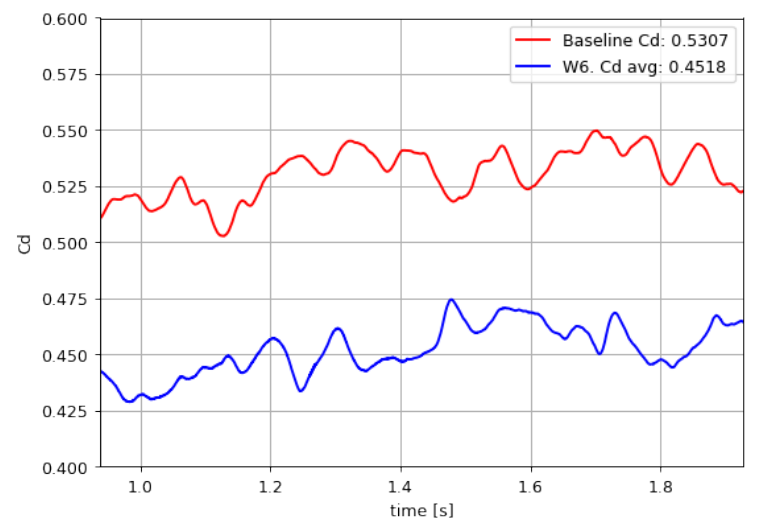

Figure 11. Comparison of drag coefficient

Figure 11 shows DDES drag coefficient history for both cases in last second of the simulation. Amplitudes of CD oscillations are similar for both cases with standard deviations of instantaneous drag coefficient being 0.0081 and 0.0095 for baseline and augmented cases respectively.

\section{Conclusions}

Two approaches for numerical modeling of flow field analyses were compared for a generic truck trailer configu- 
Table 1. Comparison of drag coefficients

\begin{tabular}{llrrr}
\hline & Component & Base & A1 & Reduction \\
\hline \multirow{3}{*}{ RANS } & Pressure & 0.4694 & 0.4415 & $5.94 \%$ \\
& Viscous & 0.0315 & 0.0301 & $4.44 \%$ \\
& Total & 0.5009 & 0.4716 & $5.85 \%$ \\
\hline \multirow{3}{*}{ DDES 1.8s } & Pressure & 0.5066 & 0.4212 & $16.86 \%$ \\
& Viscous & 0.0335 & 0.0372 & $-11.04 \%$ \\
& Total & 0.5401 & 0.4584 & $15.13 \%$ \\
\hline DDES (avg) & Total & 0.5370 & 0.4533 & $15.59 \%$
\end{tabular}

ration and augmented configuration with drag reduction devices. Results show typical for bluff body flow differences between steady RANS and DDES simulations. For a baseline case, RANS drag predictions are $6.7 \%$ lower than DDES (time averaged) results. Situation is opposite for augmented case, where RANS gives values $4.0 \%$ higher than DDES. As experimental data for given case are not available jet, it is not possible to assess deviation of both approaches from experimental flow. DDES is clearly taking into account more of the related flow physics, it therefore seems that significant amount of drag reduction potential for AFC devices is associated with inherently unsteady phenomena or non-isotropic, large scale turbulent motions not resolved in RANS approach. This questions the applicability of RANS modelling to road vehicle drag reduction studies.

\section{Acknowledgment}

This research was supported in part by PL-Grid Infrastructure.

\section{References}

[1] Reflection Group on the Future of the EU 2030, Tech. Rep. May (2010), http://www. reflectiongroup.eu/wp-content/uploads/ 2010/05/reflection $\left\{\backslash_{-}\right\}$en $\left\{\backslash_{-}\right\}$web.pdf

[2] I. Grguric, Europe 2020 - European Strategy for Smart, Sustainable and Inclusive Growth (2011)
[3] Council of the European Union, Directive (EU) 2015/719 of 29 April 2015 amending Council Directive 96/53/EC laying down for certain road vehicles circulating within the Community the maximum authorised dimensions in national and international traffic and the maximum authorised weights (2015)

[4] C. NOVAK, K. CORNELIUS, An LDV investigation of a circulation control airfoil flowfield, in 24th Aerospace Sciences Meeting (1986)

[5] K. Paschal, D. Neuhart, G. Beeler, B. Allan, Circulation Control Model Experimental Database for CFD Validation, in 50th AIAA Aerospace Sciences Meeting and Exhibit (2012), https: //ntrs . nasa.gov/ search. jsp?R=20120001334

[6] P.R. Spalart, S.R. Allmaras, A One-Equatlon Turbulence Model for Aerodynamic Flows, in AIAA paper 1992-0439 (1992), ISBN 1111111111, ISSN 00341223

[7] J. Bardina, P. Huang, T. Coakley, Turbulence Modeling Validation, Testing, and Development, NASA Tech. Memo. (1997)

[8] M.I. Yaras, A.D. Grosvenor, An Evaluation of Several Low-Re Turbulence Models Part 1- FlatPlate Boundary Layer and Axisymmetric Separating Flows, in ICAS 2002 CONGRESS (2002)

[9] F.R. Menter, Two-equation eddy-viscosity turbulence models for engineering applications (1994)

[10] D.M. Driver, Reynolds shear stress measurements in a separated boundary layer flow, in AIAA 22nd Fluid Dynamics, Plasma Dynamics and Lasers Conference, 1991 (1991)

[11] C.U. Buice, J.K. Eaton, Experimental investigation of flow through an asymmetric plane diffuser (2000)

[12] D.D. Apsley, M.A. Leschziner, Advanced turbulence modelling of separated flow in a diffuser (2000)

[13] A. Gerasimov, FLUENT: Quick Guide to Setting Up LES-type Simulations (2016), http://www . tfd. chalmers.se $\left\{\{\}\right.$ lada/comp $\left\{\backslash_{-}\right\} \operatorname{turb}\left\{\backslash_{-}\right.$ \}model/postscript $\left\{\backslash_{-}\right\}$files/Quick $\left\{\backslash_{-}\right.$ \}Guide $\left\{\backslash_{-}\right\}$to $\left\{\backslash_{-}\right\}$Setting $\left\{\backslash_{-}\right\} \operatorname{Up}\left\{\backslash_{-}\right\} \operatorname{LES}\left\{\backslash_{-}\right.$ \}version $\left\{\backslash_{-}\right\} 1.4\left\{\backslash_{-}\right\}$for $\left\{\backslash_{-}\right\}$Lars.pdf 